CAHIERS

MONDES

ANCIENS

\section{Cahiers « Mondes anciens »}

Histoire et anthropologie des mondes anciens

$11 \mid 2018$

La « civilisation " : critiques épistémologique et historique

\title{
Winckelmann et l'invention de la Grèce
}

Winckelmann and the invention of Greece

Jan Blanc

\section{(2) OpenEdition}

Journals

Édition électronique

URL : http://journals.openedition.org/mondesanciens/2089

DOI : $10.4000 /$ mondesanciens.2089

ISSN : 2107-0199

Éditeur

UMR 8210 Anthropologie et Histoire des Mondes Antiques

Référence électronique

Jan Blanc, «Winckelmann et l'invention de la Grèce », Cahiers « Mondes anciens » [En ligne], 11 | 2018,

mis en ligne le 26 mars 2018, consulté le 05 mai 2019. URL : http://journals.openedition.org/

mondesanciens/2089; DOI : 10.4000/mondesanciens.2089

Ce document a été généré automatiquement le 5 mai 2019.

\section{(c) $(1)(9)$}

Les Cahiers «Mondes Anciens " sont mis à disposition selon les termes de la licence Creative Commons Attribution - Pas d'Utilisation Commerciale - Pas de Modification 4.0 International. 


\title{
Winckelmann et l'invention de la Grèce
}

\author{
Winckelmann and the invention of Greece
}

Jan Blanc

1 Forgé dans la plupart de ses écrits, et notamment dans ses Réflexions sur l'imitation des ceuvres grecques dans la peinture et la sculpture (Gedanken über die Nachahmung der griechischen Werke in der Malerei und Bildhauerkunst, 1755) et dans sa monumentale Histoire de l'art de l'Antiquité (Geschichte der Kunst des Alterthums, 1764), Johann Joachim Winckelmann (1717-1768) n'entreprend pas de décrire l'Antiquité grecque sous l'angle exclusif d'une histoire conjoncturelle. Comme l'explique dès les premières lignes de la Geschichte, la Grèce est prise et pensée comme une totalité, comme un monde unitaire et homogène dont il s'agit de rendre compte des grands principes à travers l'étude de ses œuvres d'art:

L'histoire de l'art chez les Anciens que j'ai entrepris d'écrire n'est pas une simple relation chronologique des changements qui la constituent, car je prends le mot « histoire» dans sa signification étendue, celle qu'il a dans la langue grecque, et mon intention est de donner un essai de système doctrinal (Versuch eines Lehrgebäudes). C'est ce que j'ai tenté de réaliser dans la première partie, celle qui traite de l'art chez les peuples anciens, chez chacun d'eux en particulier, mais principalement de l'art chez les Grecs. La seconde partie comprend l'histoire de l'art au sens plus restreint, c'est-à-dire sous l'angle des circonstances extérieures, et ce, chez les Grecs et les Romains seulement. Mais l'essence de l'art (das Wesen der Kunst), dans cette seconde partie comme dans la première, reste le dessein principal (2005, p. 39-40).

2 Winckelmann présente sa vision de l'Antiquité grecque comme un système, comme une construction. Cette architecture fait prévaloir les rapports de proportion et de solidarité entre le tout et les parties, entre l'essence et l'apparence, comme l'illustre bien sa métaphore de l'« édifice doctrinal » (Lehrgebäude). Son projet est normatif et construit a priori. Il part du principe d'une «supériorité acquise par l'art des Grecs» sur celui des autres «peuples ». «L'art des Grecs est la première visée de cette Histoire et l'objet le plus digne d'être étudié et imité à cause du nombre infini de beaux monuments qu'il nous 
a légués ", explique-t-il au début du quatrième chapitre de sa Geschichte (2005, p. 224), après avoir présenté successivement, et dans l'ordre d'une progression historique et qualitative, l'art des Égyptiens, des Phéniciens, des Perses, puis des Étrusques et, enfin, des « peuples limitrophes des Étrusques » (2005, p. 100-223). Dans ce cadre, la grandeur de la Grèce répond à la manière dont elle a su civiliser les mœurs et les valeurs de l'ensemble des peuples antiques:

L'examen de l'art des Égyptiens, des Étrusques et des autres peuples peut étendre nos idées et nous aider à formuler des jugements pertinents, mais celui de l'art grec doit essayer d'unifier ces idées et d'en dégager la vérité, pour parvenir à une règle permettant le jugement et la pratique $(2005$, p. 225).

Inscrite dans un procès historique, la Grèce n'est pourtant pas approchée selon une pensée optimiste. Elle couronne en même temps qu'elle achève un cycle de progrès dans les arts et dans les mœurs, auquel a succédé un long et inexorable déclin. Ainsi, pour Winckelmann, l'art romain n'est qu'une pâle copie de l'art grec et «l'idée d'un style romain dans l'art est une illusion» $(2005$, p. 435):

Les artistes romains ne sont sans doute pas formé un style propre, mais il est probable que, dans les temps les plus reculés, ils ont imité les Étrusques, dont ils adoptèrent nombre de coutumes, notamment des rites sacrés, tandis qu'à leur époque plus tardive et florissante, leurs rares artistes ont sans doute été les élèves d'artistes grecs (2005, p. 433).

4 Et la suite de l'histoire de l'art ne peut être, à ses yeux, que le long et décevant récit d'une décadence continue, à laquelle il ne peut penser qu'avec tristesse, et que seul le retour au modèle des formes de l'art grec peut ralentir :

Le point auquel je suis parvenu dans l'histoire de l'art en dépasse déjà les limites, et bien qu'en examinant le déclin et la mort de cet art, je sois presque dans l'état d'esprit de celui qui, décrivant l'histoire de sa patrie, serait tenu d'en aborder la destruction qu'il a lui-même vécue, je n'ai pu m'empêcher de suivre le destin des œuvres d'art, aussi loin que portait ma vue. Ainsi, l'amante restée sur le rivage suit, les yeux baignés de larmes et sans espoir de le revoir, son amant qui prend la mer, et croit en voir l'image dans la voile déjà lointaine. Nous n'avons plus, comme l'amante, qu'une sorte d'ombre de l'objet de nos désirs ; mais cette silhouette nous fait d'autant plus regretter l'objet perdu, et nous examinons les copies avec bien plus d'attention que nous ne le ferions si nous avions la jouissance des originaux (2005, p. 611).

5 Contrairement à ses contemporains, Winckelmann fait donc de la Grèce non point une réalité $d u$ passé proche ou du présent, mais une valeur du passé. Elle est bien un aboutissement, mais un aboutissement révolu. Pour tenter de comprendre la manière dont est construite cette fiction de la Grèce dans les écrits de Winckelmann, j'appliquerai à sa pensée les outils de la méthode historique, en accordant une grande importance à la chronologie et en saisissant la logique structurelle d'un projet qui, avant d'être historique, est un projet moral.

\section{Le « bon goût » des Grecs}

6 «Mon œuvre principale sera l'histoire» (1952, t.I, p. 64), annonce sans ambages Winckelmann dans une lettre datée du mois d'août 1746. Il occupe alors les fonctions de précepteur, après des études de médecine à Iéna et de théologie protestante à Halle. Force, pourtant, est de constater qu'il ignore encore de quelle histoire il veut être l'auteur, ni même à quel objet il souhaiterait accorder son attention. Ce n'est que lorsqu'il 
est nommé secrétaire de la bibliothèque du comte Heinrich von Bünau (1697-1762) au château de Nöthnitz, près de Dresde, en 1748, qu'il précise la nature de son projet. Alors confronté aux 42000 volumes de cette immension collection qu'il catalogue et qu'il consulte, il développe une culture livresque considérable, notamment dans le domaine de l'histoire du Saint-Empire. Conseiller d'Auguste III de Pologne (1696-1763), électeur de Saxe (1733-1763), le comte lui permet également de découvrir les collections princières de Dresde et, en premier lieu, sa galerie de peintures, l'une des plus riches d'Europe, qui, en 1754, contient 1446 tableaux (Heres 1991, p. 69-78).

7 Ces fréquentations et ces intérêts ouvrent Winckelmann à une manière plus sensible de penser le rapport au savoir et aux archives qui, pour un temps, l'éloigne des méthodes historiques et philologiques alors en vigueur. Dans une lettre datée du 3 mars 1752, il explique que «L'érudition est une chose qui rend les hommes insensibles. [...] Ceux qui passent ici pour des savants ne connaissent que les titres et les tables des matières des livres, et c'en est assez ici pour être un savant » (1952, t. I, p. 109-110). L'histoire n'est à cet égard pensée comme une science du particulier que dans la mesure où elle subsume les exemples singuliers et les événements ponctuels sous une vision générale, comme semble l'indiquer le titre d'un brouillon datant du début de l'année 1755, les Pensées pour une contribution orale sur l'histoire générale moderne (Gedanken vom mündlichen Vortrag der neueren allgemeinen Geschichte) (Winckelmann 1968, p. 17).

8 La sensibilité de Winckelmann, toutefois, demeure alors une affaire de livres plus que d'objets. Sa connaissance des tableaux de la galerie de Dresde est incontestable ; mais elle est probablement médiatisée par les artistes qui gravitent autour d'elle, comme le peintre et graveur Matthias Österreich (1716-1778), qui travaille au Kupferstichkabinett depuis 1751 avant de devenir l'un des conservateurs de la galerie, quatre ans plus tard, ou encore le peintre et sculpteur Adam Friedrich Oeser (1717-1799), qui dispense des leçons de dessin au jeune savant. Par ailleurs, Winckelmann commence à écrire sur l'art antique alors qu'il ne le connaît que très indirectement. Considérée comme l'une des plus riches des pays germaniques, la collection électorale de marbres antiques, constituée sous le règne prestigieux de Frédéric-Auguste de Saxe (1670-1733), est conservée dans une réserve en grande partie inaccessible au public. Ce n'est que tardivement que Winckelmann y accède, en décembre 1754, quelques mois avant la publication des Gedanken. C'est la raison pour laquelle les rares statues qu'il a pu observer dans les réserves de Dresde et qu'il décrit précisément dans son essai, comme l'Agrippine ou les Vestales, ne constituent qu'une faible partie des exemples qu'il convoque (Winckelmann 1991, p. 30-32). Son savoir des œuvres antiques est d'abord nourri par les livres illustrés et par les descriptions. Aucun moulage du Laocoon n'est mentionné à Dresde durant cette période. Il ne connaît donc le groupe hellénistique qu'à travers ses lectures de la Teutsche Academie (1675-1679) de Joachim von Sandrart (1606-1688), de la Raccolta di statue antiche e moderne (1714) de Paolo Alessandro Maffei (1653-1716), ou, plus probablement encore, en consultant le De Laocoontis statua (1506) de Jacopo Sadoleto (1477-1547) et les écrits de Filippo Baldinucci (1624-1697) ou de Jonathan Richardson fils (1694-1771), qu'il recopie soigneusement dans sa bibliothèque manuscrite.

\section{Une mythographie généalogique}

9 On ne s'étonnera donc pas de voir les Gedanken, publiées en mai 1755, placées sous l'égide du mythe plutôt que de l'histoire, et cela dès les premières lignes de son opuscule : 
Le bon goût, qui se répand de plus en plus dans le monde, a commencé à se former sous le ciel grec. Les inventions des peuples étrangers ne constituèrent pour la Grèce qu'une première semence et prirent une nature et une forme différente dans ce pays que Minerve, dit-on, à cause des saisons tempérées qu'elle y avait rencontrée, avait destiné aux Grecs, de préférence à tout autre pays, car il ne pouvait manquer de produire des hommes intelligents (Winckelmann 1991, p. 15).

Winckelmann aborde la question de la Grèce sous l'angle de la mythographie. Il fait ainsi allusion au discours de Critias qui, dans le Timée de Platon, fait d'Athènes le lieu mythique d'élection d'Athéna, qui avait choisi cette terre pour «l'harmonieux mélange des saisons » qu'on y trouve et qui y rend «les hommes plus intelligents » (23c). Peut-être Winckelmann a-t-il également en tête d'autres mythes, comme ceux que Platon prête à Socrate, et qui concernent la naissance des cités grecques ou le développement des vertus civiques (Lois, III, 676a-684a ; Protagoras, 320b-322d). Il cite d'ailleurs, un peu plus loin, le célèbre larcin de Prométhée (1991, p. 57). Les premières lignes des Gedanken indiquent donc la situation du discours winckelmannien qui, à l'image des mythes grecs auxquels il se réfère comme à une origo justificatrice et fictionnelle (Dupont 2011), comportent une dimension historique, mais tout à fait incertaine. La fin qu'il affiche n'est pas, comme celle du récit historique, de dire le vrai sur le passé, mais, sur le mode du vraisemblable, de délivrer une forme de vérité sous le voile de la fable (Calame 1996 ; Calame 2000).

Cette mythographie historique portant sur la Grèce antique prend d'abord la forme d'une anthropologie physique des climats (Fink 1985). Dans la lignée de Platon, d'Aristote, mais aussi d'Hippocrate, de Polybe et, plus près de lui, de Montesquieu, Winckelmann n'hésite devant aucune schématisation: «le goût [...] s'est rarement éloigné de la Grèce sans perdre quelque chose », si bien que les « lointaines contrées » du Nord ont été en grande partie ignorées par les valeurs de cette civilisation première (1991, p. 15). Il souligne également que « l'influence d'un ciel doux et pur se faisait sentir dans la toute première éducation des Grecs » (1991, p. 17-18). Cette influence explique la beauté des corps grecs, dont témoignent les statues, si frappante quand on la compare à ceux, corrompus, des « jeunes sybarites de notre époque » (1991, p. 18).

Ajoutant que « la situation géographique particulièrement heureuse de ce pays a de tout temps été l'explication » des qualités de l'art grec (1991, p. 101), Winckelmann inscrit sa démonstration dans une perspective généalogique. C'est bien à une chaîne causale, au sens propre du terme, qu'il fait référence. Si «l'unique moyen pour nous de devenir grands et, si possible, inimitables, c'est d'imiter les Anciens » (1991, p. 16), c'est en vertu d'une proximité, d'une familiarité, voire d'une intimité avec l'art des Anciens. Il ne suffit pas d'apprendre à "connaître » et à «admirer » les " œuvres d'art de l'Antiquité, en particulier pour les Grecs » : «il faut les avoir connues comme on connaît son ami pour trouver le Laocoon aussi inimitable qu'Homère » (1991, p. 16). Le projet de Winckelmann est donc celui d'une restauration ou d'une révolution, au sens ancien du terme. Après la rupture initiée par les invasions barbares, les ténèbres du Moyen Âge et l'ignorance des Modernes, à l'exception notable de la parenthèse enchantée de la première Renaissance, il s'agit de trouver les moyens de suturer cette blessure. Imiter les Grecs, qui « veillaient à engendrer de beaux enfants» (1991, p.19), c'est tenter de devenir soi-même leur progéniture et d'enfanter à son tour une descendance digne de ce nom. 


\section{Une archéologie imaginaire}

13 De la part d'un homme qui ne s'est pas encore rendu en Italie et dont la connaissance des œuvres antiques, on l'a dit, est parcellaire et surtout livresque, cette défense et illustration de l'imitation de l'art antique peut surprendre. Il est toutefois important de souligner que, pour l'auteur des Gedanken, l'enjeu n'est nullement d'accorder une place exorbitante aux œuvres elles-mêmes, et cela pour trois raisons principales.

La première est le caractère explicitement apologétique et typologique du projet winckelmannien. Dédiées à Auguste III de Pologne, Winckelmann lui rend hommage, ainsi qu'à son père, Frédéric-Auguste de Saxe, en les plaçant tous deux dans l'exacte continuité de leurs antiques modèles homonymes:

Il faut bien reconnaître que le règne du grand Auguste est l'époque heureuse où les arts furent introduits en Saxe, alors colonie étrangère. Sous son successeur, le Titus allemand, ils furent adoptés par ce pays où, grâce à eux, le bon goût devint général. Ce sera un témoignage éternel de la grandeur de ce monarque que d'avoir exposé aux yeux de tout le monde les plus grands trésors d'Italie et les chefs-d'œuvre de la peinture des autres pays, afin de former le bon goût. Enfin, son ardeur à perpétuer les arts n'a connu aucun repos avant d'avoir fourni aux artistes de véritables et authentiques œuvres des grands maîtres grecs, et ce pour les imiter (1991, p. 15-16).

Winckelmann ne peut mieux bénir la main qui le nourrit, ni mieux faire comprendre la nature d'un projet qui prend appui sur un couple d'exempla virtutis, liés l'un à l'autre non point par une relation de contiguïté mais par un rapport de ressemblance - la Grèce des Anciens et la Saxe des Modernes : « Les plus pures sources de l'art sont ouvertes : heureux ceux qui les trouve et y goûte. Chercher ces sources signifie partir pour Athènes, et Dresde sera désormais l'Athènes des artistes » (1991, p. 15-16).

Le champ lexical de la source (arkhè) et les métaphores de l'« eau pure», que Winckelmann doit au père Dominique Bouhours (1628-1702) (Décultot 2000, p.71), indiquent un deuxième aspect de son ambition: fonder les nouvelles bases d'une " archéologie ", qu'il faut comprendre ici en son sens antique, lié à l'organisation narrative des récits mythologiques (Dupont 2011, p. 18-21; Gauvard et Sirinelli, 2015, p. 25-27). Il ne tente pas développer les principes d'une discipline scientifique consacrée à la connaissance du passé par l'étude systématique de ses traces matérielles. Il ne s'agit pas davantage, comme les sociologues et les anthropologues du début du $\mathrm{xx}^{\mathrm{e}}$ siècle (Durkheim et Mauss 1913 ; Bert 2009), de décrire la société de l'ancienne Grèce à travers ses expressions matérielles, idéologiques et religieuses. Les Gedanken proposent d'abord un grand récit, qui vise à poser et à construire, dans le passé de l'Antiquité grecque, les marques originelles d'un monde moderne que l'on peut ainsi célébrer ou critiquer, à la manière de l'archeologia mythologique :

L'archeologia mythologique n'est pas de l'histoire fabuleuse racontée jadis à de grands enfants dont la science moderne devrait rechercher aujourd'hui le noyau positif, mais des récits d'adultes destinés à fonder le présent sans se noyer dans les gouffres du passé en créant un autre temps, celui des arkhaia (les débuts). Les Anciens maîtrisaient parfaitement les deux types de récit, l'histoire événementielle et l'archeologia. Ils ne croyaient pas plus que nous à Romulus et à Mars, sachant très bien que le mythe ne doit sa vérité qu'au présent de son énonciation (Dupont 2011, p. 19).

À cet égard, et c'est un troisième aspect de sa démarche, les liens généalogiques dessinés par Winckelmann entre la Grèce antique et la Dresde moderne ne dissimulent nullement 
leur caractère fictionnel. Le savant sait bien qu'il existe une solution de continuité entre le siècle de Périclès et celui d'Auguste III. Il admet bien volontiers qu'un « retour à l'antique » est impossible puisque l'art grec a été irrémédiablement perdu par des siècles de destructions et de ruines, ce qui explique le ton souvent nostalgique de la prose de Winckelmann, comme aussi celle de Johann Wolfgang von Goethe (1749-1832), qui cite souvent son illustre prédécesseur:

Ce n'est qu'illusion quand nous souhaitons nous-mêmes habiter en permanence à Rome ou à Athènes. Il n'y a que de loin, libérée du commun et vécue réellement comme passé, que l'Antiquité nous apparaît sous des traits attirants. J'éprouve des sentiments analogues à ceux d'un de mes amis lorsqu'il regarde des ruines : nous ne pouvons être qu'irrités quand nous voyons qu'une ville à demi enfouie est dégagée, mise au jour, c'est là quelque chose qui peut tout au plus servir le savoir aux dépens de l'imagination (Goethe et Herder 1993, p. 86).

\section{Une totalité idéale} Winckelmann ne fait pas œuvre d'originalité. Comme le souligne Jeffrey Morrisson, "l'idéalisation du monde grec et de son art est l'un des aspects les plus étonnamment anhistoriques de l'œuvre théorique de la période ${ }^{1} »$. Avant lui, Anthony Ashley-Cooper, troisième comte de Shaftesbury (1671-1713), avait déjà joué un rôle essentiel dans la construction d'un discours qui, d'inspiration néo-platonicienne, faisait de l'Antiquité grecque un Âge d'or politique, moral et artistique : les statues grecques sont belles parce que les femmes et les hommes qu'elles imitaient étaient eux-mêmes beaux, et donc vrais et bons. Les notes de lecture de Winckelmann montrent qu'il connaissait ces textes. Il est un lecteur passionné des Characteristics (1711). Il cite également An Enquiry into the Life and Writings of Homer (1735), où, en connivence avec son prédécesseur, Thomas Blackwell (1701-1757), il fait d'Homère le héros d'une Grèce civilisée, qui a dépassé l'état brut de la nature avant d'avoir été corrompue par les vices de la société moderne : «On pourrait dire d'Homère et de tout poète qui écrit bien qu'il a senti et vu ce qu'il a décrit ; s'il était né plus tôt, il n'aurait vu que dénuement et barbarie ${ }^{2}$ ». Dans le sillage de ces auteurs, et selon une pratique de l'histoire encore assez courante au XVIII ${ }^{\mathrm{e}}$ siècle (Pomian 1999, p. 15-78), Winckelmann élabore les grandes périodes d'une fable grecque, qui se présente sous la forme d'une entité géographiquement et historiquement unitaire et autonome, alors qu'il n'est pas sans ignorer que son histoire est diverse et son territoire profondément hétérogène.

Comme la Grèce, l'art grec est idéalisé par Winckelmann, qui en fait le parangon de la perfection. Il admet bien certaines «négligences dans les œuvres célèbres des artistes grecs », comme le "dauphin ajouté à la Vénus Médicis ainsi que les enfants qui jouent » (1991, p. 17). Mais ce sont, dit-il, des «négligences intelligentes» (1991, p. 100), qui font partie de la grandeur même des œuvres antiques - elles obligent les spectateurs à faire la part de ce qui est superfétatoire et de ce qui est essentiel : « Les grands artistes sont sages jusque dans leurs négligences : il ne peuvent faire erreur sans instruire en même temps. Il faut regarder leurs œuvres comme Lucien affirme avoir regardé le Jupiter de Phidias, 
c'est-à-dire Jupiter lui-même et non le tabouret sur lequel reposent ses pieds » (1991, p. 17).

21 Une nouvelle fois, il s'agit de poser a priori la perfection absolue de l'art grec. Et quand les faits vont à l'encontre de la démonstration, c'est à la notion ineffable de je-ne-sais-quoi, également empruntée à Bouhours, que fait référence Winckelmann, qui donne le sentiment de sacraliser, pour ne pas dire d'adorer, les œuvres d'art grecques comme l' Apollon du Belvédère :

J'oublie tout à regarder cette merveille de l'art, je prends moi-même une noble pose pour la contempler avec dignité. Ma poitrine semble s'élargir avec respect et se soulever comme celle que je vois gonflée par l'esprit prophétique, je me sens transporté à Délos et dans les bois de Lycie qu'Apollon honora de sa présence : car mon image semble s'animer et se mettre en mouvement comme la beauté de Pygmalion. Comme la peindre et la décrire? L'art lui-même devrait m'assister et conduire ma main pour achever les traits que je viens d'ébaucher (2005, p. 556).

Écrivant ces lignes alors qu'il n'a pas encore vu la statue à Rome, Winckelmann accorde à la théorie plus d'importance qu'au regard. Sa conception du beau est, elle aussi, marquée par une puissante idéalisation de la forme et de l'intelligible, au détriment de la matière et du sensible. Il affirme que « certaines beautés idéales [...], comme l'enseigne un ancien exégète de Platon, résultent d'images élaborées par le seul entendement » (1991, p. 17) et que l'art doit parvenir "à s'étendre à des choses qui ne sont pas de l'ordre du sensible » (p. 52). Il prend ainsi exemple sur Pygmalion qui, par la seule force de son désir et de son " sentiment intérieur ", a su donner vie à une statue qu'il n'avait pas encore achevée :

C'est le sentiment intérieur qui donne à l'œuvre son caractère de vérité, et le dessinateur qui veut le donner à ses académies n'obtiendra jamais une ombre de vérité s'il ne remplace de lui-même ce que l'âme insensible et indifférente du modèle ne ressent pas et s'il n'y adjoint une attitude propre à tel sentiment ou à telle passion (1991, p. 21).

$23 \mathrm{Si}$, comme on l'a souvent noté, le projet savant de Winckelmann est de nature morale (Morrison 1996, p. 24-29), ce n'est donc pas seulement parce qu'il accorde à la Grèce antique une prééminence absolue sur tous les temps et sur tous les lieux, au nom des valeurs qu'il prétend y trouver. C'est aussi parce que la Grèce incarne d'un monde où les réalités esthétiques traduisent toujours et immanquablement des enjeux éthiques.

\section{Une éphémère réconciliation de la nature et de la culture}

Le discours nécessitariste, pour ne pas dire providentialiste, de Winckelmann exemplifie une autre dimension essentielle de sa pensée. En bon lecteur de Montesquieu, on l'a dit, il défend la théorie des climats. Il pense qu'il n'y a point de contradictions ou d'oppositions à établir entre la nature et l'idéal incarné par l'art grec, ce dernier étant le produit direct de la première: "Tout ce qui a été inspiré et enseigné par la nature et par l'art pour favoriser l'épanouissement du corps, pour le conserver, le développer et l'embellir depuis la naissance jusqu'à la pleine naissance a été mis en œuvre et employé à l'avantage de la beauté physique des Grecs anciens » (1991, p. 20). 


\section{Le pays de la « belle nature »} le pays de la « belle nature ». Peindre la Grèce ou peindre les Grecs d'après nature revient, dans cette perspective, à peindre d'après l'idéal. Les paysages de la Grèce ancienne ou le corps des Grecques et des Grecs de l'Antiquité peuvent être assimilés à la perfection dans le domaine de l'anatomie ou des proportions : « Aux Jeux d'Éleusis, Phryné se baigna sous les yeux de tous les Grecs et, au sortir de l'eau, elle fournit aux artistes l'archétype de la Vénus Anadyomène» (1991, p. 21-22). Faisant littéralement corps avec le monde qui les entoure, les Grecques et les Grecs sont eux-mêmes des morceaux de cette nature parfaite. Les hommes, plus particulièrement, veillent d'ailleurs à maintenir cette perfection par l'exercice physique, encouragé dans les gymnases et lors des Jeux Olympiques. Ils y sculptent la chair et les muscles de leur corps comme leurs statuaires travaillent le marbre de leurs statues:

Grâce à ces exercices, les corps avaient des contours grands et virils que les maîtres grecs ont donnés à leurs statues, contours sans mollesse et sans empâtement. Les jeunes Spartiates devaient, tous les dix jours, comparaître nus devant les éphores qui imposaient une diète plus sévère à ceux qui commençaient à grossier. Une loi de Pythagore imposait même de prévenir tout empâtement (1991, p. 18-19).

Les artistes grecs ne se contentent pas d'imiter la belle nature qui les entoure : « Dans les chefs-d'œuvre grecs, on trouve non seulement la plus belle nature, mais quelque chose de plus que la nature » (p. 17). Sur le modèle de Zeuxis à Crotone, ils cherchent à supprimer les quelques défauts ou les accidents qui peuvent encore être trouvés en recomposant une beauté absolument parfaite qui, de ce fait, est aussi de nature intellectuelle et suprasensible :

Ces nombreuses occasions d'observer la nature furent pour les artistes grecs l'occasion d'aller plus loin encore: ils se mirent à concevoir certaines idées générales relatives à la beauté de certaines parties du corps aussi bien qu'à l'ensemble de ses proportions, idées destinées à s'élever au-dessus de la nature ellemême et dont l'archétype était une nature spirituelle conçue dans le seul entendement (p. 22).

La Grèce ancienne est donc, et paradoxalement, un monde de la nature. Elle est, pour Winckelmann, le seul monde qui, dans l'histoire occidentale, est parvenue à s'arracher à la barbarie de la simple nature sans être pour autant touchée par la corruption des mœurs, propres aux processus inhérents à la culture. Ainsi les Grecs choisissent-ils, contre l'habitude des Modernes qui préfèrent couvrir, parer ou orner en excès leur corps, d'exalter la beauté de leurs formes et de leurs proportions en faisant le choix de vêtements aussi simples que possible :

La draperie grecque est le plus souvent travaillée d'après des vêtements fins et humides qui par suite, comme le savent les artistes, adhèrent étroitement à la peau et au corps en laissant voir le nu [...] Dans les temps modernes, on a été amené à superposer plusieurs vêtements et parfois des vêtements lourds qui ne peuvent pas retomber en plis aussi doux et ondoyants que ceux des Anciens (p. 33).

L'enjeu est bien de permettre à la beauté du corps grec de s'exprimer aussi directement et simplement que possible, sous le voile nu de la belle nature :

Le costume des Grecs était en outre conçu de façon à ne pas imposer à l'action de la nature la moindre contrainte. Le développement des belles formes ne pâtissait nullement des différentes coupes et parties de nos vêtements actuels, qui 
compriment et enserrent le corps, en particulier au cou, aux hanches et aux cuisses (p. 19).

\section{Une éthique de l'essence}

29 La beauté n'est pas, pour Winckelmann, un caractère simplement ornemental ou cosmétique de la Grèce. Elle en est, bien au contraire, sa valeur cardinale :

Quand l'artiste se fonde sur ces bases et laisse la règle grecque de la beauté conduire sa main et ses sens, il est sur la voie qui le mènera avec sûreté à l'imitation de la nature. Les notions de totalité et de perfection dans la nature antique purifieront en lui et rendront plus apparentes les notions de notre nature divisée (p. 27).

30 Deux thèmes voisins traversent ici les réflexions de Winckelmann. Le premier, déjà évoqué, est celui de l'Âge d'or, auquel l'auteur allemand donne souvent une tonalité chrétienne. La "nature divisée » dont il parle n'est pas sans rappeler la coupure symbolique instituée par le Péché originel et qu'il s'agit de réparer symboliquement, non point par le sacrifice du Christ, mais par le biais d'un retour aux valeurs et aux pratiques qui ont permis aux Grecs de l'Antiquité de fabriquer un véritable Paradis sur terre, et où la pudeur n'empêchait pas encore les jeunes gens innocents de dévoiler leur corps lors des exercices physiques : «La belle nudité des corps se montrait dans des attitudes et des positions si variées, authentiques et nobles qu'il est impossible de les faire adopter de nos jours, dans les académies, aux modèles professionnels qui sont engagés » (p. 21). La Grèce est belle parce qu'elle est bonne. C'est la grandeur d'âme qui caractérise ses artistes, aussi bien que leurs modèles, ce qu'explique le savant allemand dans son analyse du Laocoon, qui sera plus tard reprise et critiquée par Gotthold Ephraim Lessing (1729-1781) :

Laocoon ne pousse pas des cris horribles comme le Laocoon que chante Virgile: l'ouverture de la bouche ne le permet pas. Il s'agit plutôt d'un gémissement angoissé et oppressé, comme le décrit Sadolet. La douleur du corps et la grandeur de l'âme sont réparties avec la même vigueur dans l'ensemble de la figure où elles s'équilibrent. Laocoon souffre, mais souffre comme le Philoctète de Sophocle; sa détresse nous pénètre jusqu'au fond de l'âme; mais nous aimerions pouvoir supporter la détresse comme ce grand homme la supporte (p. 34-35).

31 La mesure que Winckelmann admire dans l'expression pathétique du Laocoon est aussi bien plastique que morale. Il affirme bien que « le caractère général qui distingue avant tout les chefs-d'œuvre grecs est une noble simplicité et une grandeur sereine (edle Einfalt und stille Grösse), aussi bien dans l'attitude que dans l'expression » (p. 34). Et, assurément, l'auteur des Gedanken loue la mesure avec laquelle les grands sculpteurs grecs ont su exprimer les passions les plus violentes sans tomber dans ce que le Pseudo-Longin appelle le parenthyrsis, et que Winckelmann condamne à son tour: "Toutes les actions et attitudes des figures grecques qui ne possédaient pas ce caractère de sagesse, parce qu'elles étaient trop fougueuses et trop violentes, sont tombées dans un défaut que les peintres de l'Antiquité appelaient parenthyrsis» (p.35-36). Ainsi compare-t-il deux représentations de saint Michel par Guido Reni (1575-1642) et Sebastiano Conca (1680-1764) pour donner sa préférence au premier, au nom de sa " grandeur sereine » :

Combien peu le Saint Michel du Guide, dans l'église des Capucins de Rome, a trouvé de connaisseurs capables de déceler la grandeur de l'expression que l'artiste a donnée à son archange! On déclare supérieur le Saint Michel de Conca, dont le visage exprime courroux et vengeance, alors que le Saint Michel du Guide plane sans irritation, l'air calme et serein, après avoir abattu l'ennemi de Dieu (p 39). 
Mais, comme l'a montré Élisabeth Décultot, cette théorie célèbre et devenue proverbiale n'est en réalité que la reformulation d'un topos déjà présent dans la littérature artistique $\mathrm{au} \mathrm{XVII}{ }^{e}$ siècle, qui fait l'éloge des artistes sachant donner à leurs figures des attitudes équilibrées et des proportions sans excès (Décultot 2000, p. 297). Ce qui caractérise aussi la manière dont Winckelmann conçoit et célèbre le Laocoon, en jouant sur l'ambiguïté des mots allemands, c'est la "grandeur silencieuse » (stille Grösse) d'une statue qui représente une personne capable de "souffrir en silence", c'est-à-dire en donnant à son corps la forme de son âme :

Plus l'attitude du corps est calme, plus elle est apte à exprimer le vrai caractère de l'âme: dans toutes les positions qui s'écartent trop du repos, l'âme n'est pas dans l'état qui lui est propre, elle se trouve dans un état de violence et de contrainte. C'est dans ces états de violente passion qu'elle se reconnaît le plus facilement mais, en revanche, c'est dans l'état de repos et d'harmonie qu'elle est grande et noble (1991, p. 36).

Les Grecs ressemblent à la nature dont ils sont issus. Ils sont capables de fuir l'hybris et d'aboutir à cette "tranquillité de l'âme ", cette ataraxie que leurs philosophes stoïciens ont eux-mêmes décrite et louée : « De même que les profondeurs de la mer restent calmes en tout temps, si déchaînée que soit la surface, de même l'expression dans les figures des Grecs révèle, même quand elles sont en proie aux passions les plus violentes, une âme grande et toujours égale à elle-même » (p. 34). Pour parvenir à un tel résultat, il ne suffit pas de représenter un héros stoïc qui, comme Laocoon ou Philoctète, souffre dans la solitude de son âme; il faut encore que les artistes qui représentent ces héros les comprennent en philosophes: «L'artiste a dû ressentir en lui-même la force de l'esprit qu'il imprimait à son marbre. La Grèce avait des artistes et des sages en une même personne et plus d'un Métrodore. La sagesse tendait la main à l'art et insufflait à ses figures des âmes au-dessus du commun » (p. 35).

\title{
Le monde de la pureté morale
}

Véritable Éden profane, la Grèce est le pays de la pureté morale ; mais elle est aussi celui de la pureté physique et raciale :

\begin{abstract}
La belle race des habitants de la plupart des îles grecques - bien que mélangée avec des races très différentes -, les charmes exceptionnels du beau sexe dans ces mêmes îles, en particulier dans l'île de Scios, font également conclure à la beauté des deux sexes chez des ancêtres qui se vantaient d'appartenir à une race primitive plus antique que la lune elle-même... Il existe d'ailleurs, de nos jours encore, des peuples chez lesquels la beauté n'est pas même une qualité particulière, parce que tout y est beau. Les récits de voyages sont unanimes sur ce point à propos des Géorgiens et l'on dit la même chose des Kabardiniens, peuplade tartare de Crimée (p. 20).
\end{abstract}

Alors que, malgré sa théorie des climats, Montesquieu affirme l'unité de l'espèce humaine (Colas 2004, p. 190-204), Winckelmann suit ici les idées de Voltaire, pour lequel il a existé et il existe toujours « différentes races d'hommes » (Voltaire 1805, p. 210), dans le but de parachever sa démonstration d'un caractère naturel de la civilisation grecque. Il ne suffit pas de démontrer que la Grèce est le monde de la belle nature. Il faut encore convaincre que les Grecs eux-mêmes en sont les œuvres. Winckelmann insiste donc sur le caractère idéal du corps grec qui, contrairement à celui des autres nations, présentent les caractéristiques formelles susceptibles de le réduire à des formes géométriques simples, comme c'est le cas, par exemple, de la ligne continue du front et du nez : « Chez les dieux et les déesses, le front et le nez formaient presque une ligne droite » (1991, p. 22). 
La pureté des corps grecs ne se traduit d'ailleurs pas seulement par la perfection de leurs formes, mais aussi par la plénitude de leur santé, seule garantie du maintien de cette perfection, à travers les générations, et par-delà le temps, qui, dans toute autre circonstance, aurait tôt fait de la corrompre et de la détruire :

Les maladies qui détruisent tant de beautés et défigurent les corps les plus parfaits étaient encore inconnues chez les Grecs. Nulle part, dans les écrits des médecins grecs, n'est mentionnée la variole, dans aucune des descriptions si détaillées que fait Homère des corps grecs ne se trouve de signe, pourtant bien caractéristique, de la petite vérole. Les maladies vénériennes et le rachitisme qui en découle n'exerçaient pas non plus leurs ravages sur la beauté naturelle des Grecs (p. 20).

Ces analyses morales et naturalistes de la civilisation grecque sont d'autant plus compréhensibles et facilitées que Winckelmann n'a, au moment où il rédige ses Gedanken, qu'une connaissance fort limitée de l'art et des œuvres antiques. Une grande partie des exemples sur lesquels il s'appuie, et parfois longuement, comme c'est le cas du Laocoon, ne lui sont connus que par des interprétations - moulages, estampes, descriptions. Comme le savant allemand le reconnaît lui-même, il n'a pas encore su donner à sa conception de la Grèce les contours d'une véritable histoire, fondée sur l'analyse empirique et matérielle des objets: "Ma conviction est que la beauté en art tient davantage à la finesse de la sensibilité et à la pureté du goût qu'à une réflexion approfondie » (p. 99).

La « conviction » de Winckelmann, nourrie d'une lecture idéaliste de la Grèce ancienne, change pourtant assez radicalement après son arrivée à Rome. On comprend, de ce fait, les hésitations marquées par le savant devant l'explication événementielle de l'art grec. Puisque que la période qui l'incarne le mieux, le $v^{\mathrm{e}}$ siècle avant notre ère, constitue un moment exceptionnel mais aussi passager de la grandeur antique, il paraît difficile à Winckelmann de l'inscrire dans un système explicatif trop étroit, qui pourrait donner le sentiment illusoire qu'il suffirait de réunir les mêmes causes pour produire les mêmes effets. Parce qu'il peut être considéré, en ce sens, comme le père de la notion de «miracle grec $^{3}$ ", Winckelmann résiste encore, avant de quitter Dresde pour Rome, à l'idée qu'une histoire froide et objective de l'art grec est possible et même souhaitable.

\section{Un antidote aux Modernes}

Grâce à une pension de 200 thalers d'Auguste III, Winckelmann finit par fouler le sol de Rome en novembre 1755. Et, un mois plus tard, seulement, il admet qu'il doit entièrement revoir son projet et ses idées: "J'ai déjà appris qu'on parle en myope des antiquités, d'après des livres, mais sans les avoir vues; j'ai découvert moi-même des erreurs que j'avais commises" (1952, t. I, p. 191). La carrière romaine de Winckelmann demeure toujours attachée au monde des livres. Bibliothécaire des cardinaux Domenico Silvio Passionei (1682-1761), Alberico Archinto (1698-1758) et Alessandro Albani (1693-1779), il est ensuite nommé libraire et scriptor de la bibliothèque Vaticane. Pour autant, ces activités lui permettent, bien plus qu'à Dresde, de se retrouver au contact des objets et des artistes. Il analyse chacune des pièces antiques auxquelles il peut avoir accès dans les extraordinaires collections romaines. Il travaille de façon systématique, allant jusqu'à produire des descriptions extrêmement précises des antiques qui l'intéressent le plus, dans la cour du Belvédère, comme l'Apollon, le Torse, l'Antinoüs ou le Laocoon. Il se rend à Naples et visite les fouilles d'Herculanum et de Pompéi, ainsi que le palais royal de Portici. Sa fréquentation des peintres et des sculpteurs, notamment dans l'entourage du cardinal 
Albani, à l'instar de son compatriote Anton Raphaël Mengs (1728-1779), avec lequel il vit un temps, lui permet également de raffiner son rapport aux œuvres en tenant compte des enjeux techniques et des problèmes matériels d'exécution et de conservation.

Ces expériences permettent à Winckelmann de devenir en quelques années l'un des antiquaires les plus respectés de Rome et lui valent d'être nommé préfet des antiquités par Clément XIII, en 1763. Il met alors la dernière main à un nouvel ouvrage, la Geschichte der Kunst des Alterthums, parue en allemand et à Dresde en 1764, et dédié à Frédéric IV de Saxe (1722-1763). Ce dernier vient tout juste d'accéder à la fonction électorale, après la mort de son père Auguste III, le 5 octobre 1763. L'ambition affichée par Winckelmann, plus monumentale que celle des Gedanken, est de proposer une « histoire » (Geschichte) de l'art antique, même si, une nouvelle fois, la méthode proposée mérite d'être questionnée.

\section{Téléologie et eschatologie}

41 Cette dédicace est d'une toute autre nature que celle qui ouvrait les Gedanken. Contrairement à Auguste III, auquel Winckelmann était attaché et obligé, Frédéric IV n'est pas le mécène du savant allemand, qui est désormais employé par le Saint-Siège, après une conversion au catholicisme probablement mue par ses ambitions personnelles. C'est donc au prince dont il est le sujet que Winckelmann s'adresse. Il le remercie d'avoir "gracieusement daigné accepter» les "prémices de [ses] travaux romains en langue allemande ", loue non sans une certaine malice " un profond connaisseur », " en vertu de la connaissance qu'Elle a acquise à travers la contemplation des œuvres de l'art ancien et moderne pendant une année entière à Rome ». Et il souligne que cette Geschichte présente « des fruits artistiques plus mûrs » dont la croissance doit moins à l'activité bénéfique de l'Électeur qu'au climat romain : "premiers dans leur genre, [ils] ont grandi au sein de l'Antiquité et des arts et se sont nourris et perfectionnés sous ce ciel qui m'est propice » (2005, p. 36).

Winckelmann n'a donc pas renoncé à l'essentiel de ses idées. S'appliquant à lui-même sa théorie des climats, il constate que le succès de son entreprise doit beaucoup au « ciel » romain. S'il n'a pas la pureté de celui de la Grèce antique, il s'en rapproche fortement et lui permet de renforcer ses hypothèses, en commençant, précisément, par proposer une périodisation plus fine de l'art antique. Il demeure attaché à l'idée selon laquelle l'art grec a constitué, dans l'histoire des arts anciens et modernes, un summum indépassable. Mais ce lecteur de L'Antiquité expliquée et représentée en figures $(1719,10$ vol.) de Bernard de Montfaucon (1655-1741) et du Recueil des Antiquités égyptiennes, étrusques, grecques et romaines (1752-1767, 7 vol.) du comte de Caylus (1692-1765), qui insiste sur la primauté de l'art égyptien (1751, t. I, p.117-118), est conscient de la double nécessité de mieux distinguer l'art grec de l'art romain et de donner des gages supplémentaires à la supériorité du premier sur les autres arts de l'Antiquité.

Pour cela, Winckelmann propose pour la première fois une périodisation de l'art antique. Par définition, cette périodisation procède d'une logique téléologique, qui lui permet désormais de faire de l'art grec le sommet d'un mouvement historique :

Le style le plus ancien a duré jusqu'à Phidias ; c'est grâce à lui et aux artistes de son époque que l'art parvient à la grandeur, et ce style peut être appelé le grand style ou le haut style ; de Praxitèle ou Lysippe et à Apelle, l'art acquit plus de grâce et de séduction, et ce style devrait être appelé le beau style. Quelque temps après ces artistes et leur école, l'art commença à décliner avec les imitateurs - et nous 
pourrions alors parler d'un troisième style, celui des imitateurs - jusqu'à ce qu'il

tendît progressivement à sa chute (2005, p. 333-334).

d depuis les Gedanken, la grandeur suprême de l'art grec, pour la célébration de laquelle l'ensemble des outils et des méthodes historiques doivent être mobilisés. Mais elle est également eschatologique, dans la mesure où le "grand style » est irrémédiablement perdu. L'avis de Winckelmann n'est pas partagé par tous ses contemporains. Lors de son voyage italien, en 1752, le capitaine anglais John Northall, officier de l'armée britannique et écrivain, décrit le sentiment qu'il ressent, dans les marbres antiques, d'un voyage dans le temps et dans l'espace: "Voir des empereurs, consuls, généraux, orateurs, philosophes, poètes et autres grands hommes dont la renommée a depuis longtemps attiré notre attention, se tenir là en personne devant nous, renvoie un homme deux mille ans en arrière et mêle le passé au présent » (Haskell 1988, p. 68). Plusieurs décennies plus tard, le peintre anglais Joshua Reynolds (1723-1792) utilise les mêmes métaphores pour louer les peintures d'histoire et les paysages de Nicolas Poussin (1594-1665), de Claude Lorrain (1600-1682) ou de Sébastien Bourdon (1616-1671), capables de restituer l'expérience du monde antique :

Poussin semble penser que le style et le langage dans lesquels de telles histoires sont racontées ne perdent rien à conserver quelque reste de l'ancienne façon de peindre qui, pour lui, consiste en une certaine uniformité générale du tout. Cette 
manière permet à l'esprit de se transporter dans l'antiquité par le sujet mais aussi par l'exécution. [...] À l'instar de Nicolas Poussin, [le bon peintre] transportera le spectateur dans les environs de la Rome ancienne, en nous montrant tous les objets qu'une éducation littéraire nous rend si précieux et si intéressants, ou, comme Sébastien Bourdon, nous plongera dans les antiques ténèbres des pyramides d'Égypte, ou encore, comme Claude Lorrain, nous conduira vers la tranquillité des scènes arcadiennes et des terres enchantées. [...] Il faut que le caractère du paysage, et cela dans toutes ses parties, soit adapté à la représentation historique ou poétique. C'est une entreprise très difficile, qui demande de transporter son esprit deux mille ans en arrière, de la naturaliser pour ainsi dire dans l'antiquité, comme c'était le cas de celui de Poussin (Blanc 2015, p. 445, 828, 861).

Le constat de Winckelmann est assez différent. La beauté de l'art grec appartient au passé et ne peut qu'y demeurer. Elle ne peut, au mieux, que susciter le lamento pathétique de ses amateurs, comme celui du savant allemand, dans sa Description $d u$ Torse du Belvédère, $\grave{a}$ Rome (Beschreibung des Torso in Belvedere zu Rom, 1762) :

Je reste saisi de tristesse, et de même que Psyché commença à pleurer l'Amour après l'avoir connu, de même je déplore l'irrémédiable altération de cet Hercule, après être parvenu à en percevoir la beauté. L'art pleure avec moi ; car cette œuvre, qu'il pouvait opposer aux plus grandes découvertes de l'esprit et de la réflexion, et grâce à laquelle il pouvait, comme à l'âge d'or, s'élever au sommet de l'admiration humaine; cette œuvre qui est peut-être la dernière à laquelle il a appliqué toutes ses forces, cette œuvre, il faut qu'il la voie maintenant à moitié détruite et cruellement maltraitée (Décultot 2000, p. 276-277).

Puisqu'elle est inatteignable, la beauté du « grand style » grec ne peut donc faire l'objet d'une imitation directe. Jamais la copie ne pourrait atteindre la perfection de l'original. C'est pourquoi il condamne l'émulation défendue par le Bernin, qui exprime l'orgueil d'un sculpteur moderne qui ne sait plus ce qu'il doit à la statuaire antique :

On sait que le célèbre Bernin fut l'un de ceux qui voulaient contester aux Grecs et l'avantage d'une nature plus belle, et la beauté idéale de leurs figures. Il estimait en outre que la nature savait accorder à toutes ses parties la beauté nécessaire - l'art consistant, selon lui, à trouver cette beauté. Il se vanta de s'être défait d'un préjugé qu'il éprouvait au début en contemplant le charme de la Vénus Médicis : ce charme, il prétendit plus tard l'avoir découvert dans la nature en diverses occasions, au terme d'une laborieuse étude. C'est donc cette Vénus qui lui apprit à découvrir dans la nature des beautés qu'il croyait tout d'abord ne pouvoir trouver que dans cette statue (1991, p. 25).

Face à l'antique, seules deux approches sont envisageables. Contrairement à ce que l'on a souvent affirmé, il ne prône pas un "retour à l'antique", parce que ce retour est impossible (Michel 2010). Le savant encourage donc les artistes à s'inspirer du bon goût des artistes grecs plutôt qu'à en copier littéralement les œuvres. Il propose de distinguer ces deux opérations dans son Souvenir sur la contemplation des œuvres d'art (Erinnerung über die Betrachtung der Werke der Kunst, 1759) :

À la pensée personnelle, j'oppose la copie (das Nachmachen), mais nullement l'imitation (die Nachahmung): sous le terme de copie, j'entends le calque servile ( knechtische Folge). Dans l'imitation, au contraire, l'objet imité, lorsqu'il l'est avec intelligence, peut devenir pour ainsi dire une nouvelle nature et constituer quelque chose d'autonome (Décultot 2000, p. 110).

Mais les hommes de lettres et les historiens peuvent, eux aussi, participer de la célébration de l'art grec. S'il explique bien, dans une lettre du 20 mars 1756, que « la description de l'Apollon [du Belvédère] demande un style sublime, qui s'élève au-dessus de tout ce qui est humain » mais qu'on « ne peut pas décrire l'effet que provoque l'apparence 
de cette œuvre » (1952, t. I, p. 212), Winckelmann encourage les premiers à faire de leur mieux et, par la pratique de la description, à tenter de restituer les sentiments et les effets que l'observation des œuvres antiques ont produits sur leurs sens et leur imagination. Par la puissance des mots et du langage, il s'agit pas de ressusciter la beauté de l'art grec, mais de reconstruire les conditions dans lesquelles elle peut encore être appréciée par les Modernes.

\section{Centre et périphérie}

51 Winckelmann propose donc une périodisation plus précise de l'art antique, orientée vers la glorification de l'apothéose grecque, mais encore une nouvelle géographie des arts antiques, dont le centre de gravité est la Grèce et, plus précisément, l'Attique. Dans les Gedanken, il avait déjà tenté d'établir quelques nuances entre les différentes régions de la Grande Grèce, en opposant à la vertueuse fermeté politique des Athéniens l'amollissement moral des Grecs d'Orient (Pommier 2003, p. 193). Ce n'est toutefois qu'à partir de la Geschichte qu'il jette les bases d'un nouveau regard sur les espaces de l'art antique qui, comme toutes les géographies artistiques, repose sur des présupposés idéologiques particulièrement forts ${ }^{4}$.

La périodisation proposée par Winckelmann procède de la volonté de placer au cœur de l'histoire le "grand style " grec du $\mathrm{v}^{\mathrm{e}}$ siècle. Sa géographie, de son côté, envisage une modélisation essentiellement symbolique de l'espace de la Grande Grèce. Au cœur de ce territoire, l'Attique occupe la place privilégiée d'un centre qui réunit toutes les qualités idéales :

La région de l'Attique, comme autrefois, est l'image même de l'amour des gens. Tous les bergers et tous ceux qui travaillent aux champs ont accueilli à bras ouverts les deux voyageurs [Jacob] Spon [1647-1685] et [George] Wheler [1651-1724], sont allés à leur rencontre en leur souhaitant la bienvenue (1991, p. 109).

Les artistes et les œuvres issus des cités voisines, comme Corinthe ou Sicyone, sont également associés à la grandeur du pays d'Athènes, ainsi que, pour des raisons liées à leur prestige ancien, les villes d'Éphèse et de Rhodes. Elles ne sont toutefois que des exceptions. Pourtant située à égale distance d'Athènes que Corinthe, Thèbes est déjà considérée comme une cité dont l'âme grecque est corrompue, essentiellement pour des raisons climatiques : elle était « habitée par un ciel lourd et ses habitants étaient lourds et robustes ", tandis que « la région de l'Attique bénéficiait d'un ciel pur et serein qui a eu pour conséquence une profonde acuité des sens (que l'on impute aux Athéniens) et qui a façonné ces corps parfaitement proportionnés. Et c'est à Athènes que se trouvait le centre éminent des arts »(p.107). Pour les mêmes raisons, Sparte est visée par Winckelmann qui, se faisant le relais direct du discours de Périclès, explique que le modèle politique lacédémonien, « fondamentalement différent de celui du reste de la Grèce », a produit des corps et des artistes différents : «Les Spartiates, dit Périclès, cherchent dès leur jeunesse à acquérir la force virile par la pratique d'exercices violents. [...] Nous aimons la délicatesse, sans abus, et la sagesse, sans mollesse » (p. 106).

Pour le reste, « les colonies [grecques] à l'étranger ont connu pratiquement le sort de l'éloquence grecque dès qu'elle sortait de ses frontières » (p. 107-108) : elles ont subi une corruption des mœurs et des arts proportionnelle à la distance à laquelle elle se trouvait d'Athènes. Quant aux arts non grecs, comme les arts étrusques ou égyptiens, leur primauté, pourtant admise depuis Pline l'Ancien, est clairement remise en cause par 
Winckelmann, qui constate que, dans cette "prétendue patrie des arts et des sciences", «quantité d'effets de la nature » sont "entravés par des lois sévères », et où «les plus parfaites créatures de la nature n'auraient pu être connues qu'imparfaitement par les artistes » (p. 20-21). Contrairement à l'Égypte, « en Grèce, [...] où l'on s'adonnait dès sa jeunesse au plaisir et à la joie, où le "savoir-vivre" bourgeois ne faisait pas obstacle comme aujourd'hui à la liberté des mœurs, la belle nature se montrait sans voiles pour le plus grand profit des artistes » (p. 21).

\section{Modernes et Anciens}

55 La géographie artistique théorisée par Winckelmann, qui oppose le centre grec à ses périphéries, schématise donc une dichotomie et oppose la grandeur de la Grèce à toutes les autres sociétés antiques méditerranéennes. Cette description apparait conditionnée par les critiques qu'il ne cesse de formuler à l'égard du monde contemporain. Les idées du savant allemand se rapprochent en cela de celles de Jean-Jacques Rousseau (1712-1778), auquel Denis Diderot (1713-1784) le compare dès 1765 :

Tel est Jean-Jacques Rousseau lorsqu'il se déchaîne contre les lettres qu'il a cultivées toute sa vie, la philosophie qu'il professe, la société de nos villes corrompues au milieu desquelles il brûle d'habiter et où il serait désespéré d'être ignoré, méconnu, oublié. [...] Tel est Winckelmann lorsqu'il compare les productions des artistes anciens et celles des artistes modernes (Diderot 2000, p. 438-439, Salon de 1765).

Comme l'auteur de l'Émile, Winckelmann paraît prendre le parti des antimodernes. Il dénonce la modernité au nom d'une autre conception de la modernité, faisant de la société moderne une communauté artificielle qui, organisant de façon excessivement rationnelle les rapports sociaux, met à distance les vérités authentiques de la nature humaine (Compagnon 2005). Ces principes guident les reproches que Winckelmann adresse aux sculpteurs et aux peintres contemporains et, plus largement, aux artistes qui, depuis la Renaissance, n'ont pas su ou pas voulu prendre l'antique pour modèle principal. Sans doute est-ce à Rome que l'auteur allemand découvre, au contact rapproché des objets, que la sculpture antique était polychrome. Mais, tout comme Joshua Reynolds, il fait comme si les statues grecques et romaines étaient blanches, au nom de la qualité intellectuelle que cette couleur blanche, "neutre", confère aux œuvres qu'il veut célébrer pour leur beauté idéale (Blanc 2016; Brinkmann et Kunze 2011). C'est la raison préjudicielle pour laquelle Winckelmann n'accorde guère d'importance à la peinture ancienne. Il cite les Noces Aldobrandines et les fresques d'Herculanum comme des œuvres importantes (1991, p. 48-49) ; mais il reconnaît aussi que les « peintres grecs » n'ont pas tout inventé. Il leur accorde « l'art du dessin et de l'expression, mais sans plus : on leur dénie l'art de la perspective, de la composition et du coloris » (p.48), des domaines dans lesquels, de son aveu même, la peinture des Modernes a été supérieure à celle des Anciens (p. 50). Mais cette concession permet à Winckelmann de conserver l'essentiel, en réactivant notamment les débats anciens qui ont agité les théories artistiques européennes depuis le début de la Renaissance et en s'affirmant comme un farouche partisan de la primauté du dessin et de la ligne contre les «fards» de la couleur (Lichtenstein 1989) :

La couleur contribue à la beauté, mais elle n'est pas la beauté même: elle la rehausse, elle et ses formes. Comme le blanc est la couleur qui réfléchit le plus grand nombre de rayons lumineux, c'est aussi la plus sensible, et un beau corps sera d'autant plus beau qu'il est plus blanc et, s'il est nu, paraîtra plus grand qu'il n'est 
en réalité, comme nous voyons que tous les moulages modernes en plâtre paraissent plus grands que les statues originales (2005, p. 244). argument. Peter Paul Rubens (1577-1640) est une cible facile. Il incarne, pour l'auteur allemand, le goût moderne pour les grimaces et les attitudes trop recherchées et le refus des proportions canoniques adoptées par les artistes antiques, symboles d'une société factive qui cultive le monde des apparences et des faux-semblants. Rubens est toutefois moins mauvais que son compatriote Jacob Jordaens (1593-1678), par exemple (1991, p. 118), puisque, bien qu'il soit "bien éloigné du contour des corps grecs», il a eu le mérite de l'amender après «avoir étudié les Anciens» (p. 29). Le Bernin (1598-1680) est également l'un des sculpteurs contre lesquels Winckelmann concentre de nombreuses attaques, lui reprochant d'avoir introduit «dans l'art une corruption qui dure encore » (2005, p. 362) mais aussi les critiques qu'il adresse à la statuaire grecque (p. 251).

Cette corruption est d'abord celle des « œuvres de la majorité des artistes modernes » qui, plutôt que d'imiter « la sagesse dans l'expression dont firent preuve les artistes anciens ", "n'expriment pas beaucoup avec peu, mais peu avec beaucoup», ressemblant en cela « aux comédiens des théâtres antiques, obligés d'outrer la vérité au-delà de ses limites pour se faire comprendre, en plein jour, de n'importe quel spectateur de la plèbe placà au dernier rang» (p. 278). Elle fait donc prévaloir le superflu sur le nécessaire, l'accidentel sur l'essentiel, qui, au moment où Winckelmann écrit ces lignes, lui paraît dominer l'essentiel de la production contemporaine, et à laquelle il ne voit d'autre antidote que l'imitation plus fidèle des statues antiques et un attachement plus intense aux efforts de symbolisation des artistes grecs: "Nos volutes et nos chères rocailles, sans lesquelles aucun ornement ne peut se faire aujourd'hui, n'ont souvent guère plus de naturel que les candélabres de Vitruve qui supportaient de petits châteaux et palais. L'allégorie pourrait apporter l'érudition nécessaire qui apprendrait à accorder le plus petit ornement à la place qu'il occupe » (1991, p. 55). Pour résumer, l'art doit être, pour Winckelmann, le règne du vraisemblable et de l'universel plutôt que du vrai et du singulier :

L'imitation du beau dans la nature ou bien vise un objet particulier, ou bien rassemble les remarques inspirées par différents objets pour en faire une synthèse. La première démarche consiste à faire une copie ressemblante, un portrait, c'est la voie qui conduit aux formes et figures des Hollandais. La seconde est la voie qui mène au beau universel et à ses images idéales : c'est la voie qu'ont suivie les Grecs (p. 26).

Certains artistes modernes sont parvenus à cette perfection, mais seulement dans certaines classes de figures. Ainsi en est-t-il de Michel-Ange, dont l'idéal personnel brille dans ses représentations de nus masculins, mais qui le rend incapable d'être à la hauteur de la beauté des nus féminins antiques :

Michel-Ange est peut-être le seul dont on puisse dire qu'il a atteint le niveau des Anciens, mais uniquement dans le cas des figures fortement musclées des corps de l'époque héroïque. Il n'y est pas parvenu, par contre, dans les figures délicates et jeunes ni dans les figures féminines qui sont devenues, sous sa main, des amazones (p. 29).

Au-delà des incorrections de ces artistes, c'est ainsi au charme immédiat qu'ils tentent de susciter auprès de leurs spectateurs que s'attaque Winckelmann. Sur le modèle de la «beauté grecque» ou de la Madone Sixtine de Raphaël (1483-1520), le savant ne croit qu'aux mérites d'un art qui fait le choix de sacrifier une certaine manière de séduire au profit d'une plus grande formalité : « Ses tableaux auraient pu avoir plus de diversité, des 
vêtements plus amples, plus de coloris, plus de lumière et d'ombre, mais ses figures auraient toujours eu moins de valeur que celle que lui ont conférée les nobles contours et l'âme sublime que les Grecs lui avaient appris à représenter » (p. 28).

61 Si la Geschichte de Winckelmann affirme donc un projet historique dont les contours sont bien mieux dessinés que ceux des Gedanken, cette "histoire » est, de l'aveu même du savant allemand, innervée par le sentiment nostalgique d'une perte irrémédiable mais aussi d'une corruption généralisée des mœurs et des arts du XVIII ${ }^{e}$ siècle. À cette décadence, consubstantielle à la modernité, Winckelmann opposera l'antidote de l'art grec qui, s'il ne permettra pas aux hommes de pouvoir entièrement guérir de leur maladie, leur offrira la possibilité d'un salut temporaire.

\section{Conclusion}

Les écrits du savant et antiquaire allemand Johann Joachim Winckelmann (1717-1768) n'emploient jamais le mot « civilisation ». Ce terme n'apparaît qu'à partir de la deuxième moitié du XVIII ${ }^{\mathrm{e}}$ siècle, d'abord dans la langue française, avant de se diffuser en Europe. C'est probablement à Victor Riquetti de Mirabeau (1715-1789), le père du célèbre révolutionnaire, que l'on doit le premier usage de ce néologisme. Dans L'Ami des Hommes, ou Traité de la population (1755), il affirme ainsi que «la religion est sans contredit le premier et le plus utile frein de l'humanité : c'est le premier ressort de la civilisation » (1755, p. 192).

63 Les premiers usages du terme civilisation montrent alors une certaine ambivalence. Dérivé de l'adjectif civil (XIII ${ }^{\text {e }}$ siècle) et du substantif civilisé (XIv ${ }^{\mathrm{e}}$ siècle), lui-même emprunté au latin (civis, civitas, civilis), la civilisation désigne en premier lieu le processus par lequel une société, un monde ou une période se civilise ou est civilisé, c'est-à-dire se rapproche de la civilité, entendue comme une "manière honnête, douce et polie d'agir, de converser ensemble (Furetière, 1690)». Dès le $\mathrm{XVI}^{\mathrm{e}}$ siècle, «civiliser» revient à «mener à la civilité ", à "rendre civiles et douces les mœurs et les manières des individus " (Starobinski 1983, p. 13). Dans De l'Esprit des Lois (1748), Charles Louis de Secondat de Montesquieu (1689-1755) évoque la manière dont Alexandre le Grand « fit creuser des puits, bâtir des villes » en Inde, et « défendit aux Ichthyophages de vivre de poisson » car «il voulait que les bords de cette mer fussent habités par des nations civilisées » (1838, p. 362-363, IV, XXI, 8). En 1767, le philosophe et historien écossais Adam Ferguson (1723-1816) explique encore que «tout comme les individus avancent en passant de l'enfance à l'âge adulte, leur espèce même avance en passant de la rudesse à la civilisation ${ }^{5}$ ». Mais, comme l'indique la cinquième édition du Dictionnaire de l'Académie (1798), la civilisation désigne aussi l'« état de ce qui est civilisé » (Starobinski 1983, p. 14). Dans ses Éphémérides du citoyen (1767), Mirabeau fait également référence à ce sens. Il remarque que «les richesses mobiliaires d'une nation dépendent donc non seulement de sa civilisation, mais encore de celle de ses voisins » (Starobinski 1983, p. 19).

Pour les raisons évoquées dans cet article, les écrits de Winckelmann me semblent toutefois participer du contexte intellectuel et philosophique dans lequel la notion de « civilisation » a été forgée. historiens, historiens de l'art et archéologues du xix ${ }^{e}$ et du xx siècles, Winckelmann peut 
être considéré comme le premier historien de l'art. C'est notamment l'avis exprimé par Aloïs Riegl (1858-1905) :

Ce qui fait de Winckelmann le premier historien d'art, c'est son penchant prononcé à déterminer et mettre en évidence les traits communs dont il a découvert la présence dans toutes les œuvres de l'Antiquité qu'il a étudiées. Ce qui l'intéressait, ce n'était pas l'existence singulière de l'œuvre d'art considérée pour elle-même, mais plutôt ce trait commun qui met en relation toutes les œuvres singulières, les unissant pour composer un ensemble supérieur, même si cette unité ne se réalise qu'au niveau conceptuel (Kultermann 1993, p. 54).

Or il est essentiel de souligner que l'opinion de Riegl ne peut être pertinente que s'il est réinscrite dans une vision de l'histoire qui, elle-même héritée des conceptions du xvIII ${ }^{\mathrm{e}}$ siècle, et réactualisée au $\mathrm{XIX}^{\mathrm{e}}$ siècle par les grands monuments de Jules Michelet, prétendait pouvoir et devoir faire émerger de la pluralité chaotique des événements les grandes lois et les principales étapes de l'Esprit en marche. En imaginant écrire ne seraitce qu'une Introduction à l'Histoire universelle (1831) ou rendre compte, par les moyens de l'histoire, d'une notion aussi générale et contestable que Le Peuple (1846), l'historien français a sans doute le mieux incarné, au XIX ${ }^{e}$ siècle, cette conception post-hégélienne d'une histoire qui fait le récit de la "liberté humaine». Le particulier y est toujours subsumé sous le général ; et l'essentiel de la tâche de l'histoire est de décrire et d'analyser les grandes civilisations du passé plutôt que de se perdre dans les événements, les individus et les cas d'étude. Une idée qui, plus tard, et selon des modalités différentes, a été reprise à son compte par Arnold Toynbee (1889-1975), ou encore, au lendemain de la Seconde Guerre Mondiale, par les historiens des Annales, une revue créée en 1929 et qui change alors de titre pour s'appeler Annales. Économies, Sociétés, Civilisations. Il est d'ailleurs significatif qu'après avoir publié ainsi un long article intitulé "Civilisation: évolution d'un mot et d'un groupe d'idées» (Febvre, Mauss, Tonnelat et Niceforo 1930), Lucien Febvre ait été le premier occupant de la chaire d'Histoire de la civilisation moderne, créée au Collège de France en 1933, avant que ne lui succède Fernand Braudel (1950-1972), dont la deuxième partie de La Méditerranée et le monde méditerranéen à l'époque de Philippe II explique vouloir étudier «successivement les économies et les États, les sociétés, les civilisations » (Braudel 1990, p. 13).

Si nous nous fondons sur l'histoire telle qu'elle est pensée et écrite aujourd'hui, Winckelmann ne peut et ne devrait donc être considéré comme le premier historien ou le premier historien de l'art moderne. Postulant qu'il existe un monde grec, cohérent par la manière dont il articule les événements et les productions de l'esprit, le savant allemand porte sur lui un jugement d'abord moral, qui l'amène à la considérer comme la traduction la plus pure de la belle nature mais aussi à orienter son discours et ses démonstrations. Son projet est pris dans un moment essentiel de l'histoire de l'histoire, où le goût jadis cultivé pour les belles fables et les grands récits est progressivement contesté par une nouvelle conception de la vérité, fondée sur l'examen critique des sources et le refus des ornements de la belle langue (Flavell 1979 ; Howard 1990 ; Potts 1982 et 1990 ; Seeba 1982).

Tous les lecteurs contemporains de Winckelmann ne se sont point agacés des approximations, voire de ses nombreuses erreurs de chronologie, d'attribution et d'identification, souvent dictées par la volonté de faire prévaloir la validité de son modèle d'explication général contre la résistance des faits et des objets. Ainsi, l'Apollon du Belvédère, copie romaine en marbre réalisée à l'époque d'Hadrien d'un ancien bronze de Léocharès, datant de 330-320 avant Jésus-Christ, et réalisé pour l'agora d'Athènes, doit nécessairement provenir du temple de Delphes et constituer «le plus haut idéal de l'art 
parmi toutes les œuvres antiques qui ont échappé à la destruction ", tant il occupe une place stratégique dans la démonstration de Winckelmann. Le nom de Léocharès n'est certes pas inconnu de Winckelmann, qui le place parmi la foule anonyme des suiveurs de Canachos, avec Bryaxis et Timothée (2005, p. 491), et ne lui attribue toutefois qu'une seule œuvre, un Ganymède alors à la villa Médicis (auj. Florence, Museo Archeologico), qui est lui-même, en réalité, une sculpture moderne (Kaibel 1890, p. 324)... Sans doute ces flottements passent-ils inaperçus aux yeux des contemporains de Winckelmann, tandis que ceux qui sont capables de les détecter n'y voient que les détails ou les contreparties nécessaires d'un discours qui, pour être authentique, doit se détacher de la stricte et plate vérité historique.

Pour d'autres lecteurs de Winckelmann, en revanche, y compris pour ses amis ou ses défenseurs, ce rapport problématique à la vérité n'est pas acceptable. C'est le cas de Herder, qui rappelle que de nombreux connaisseurs ont relevé les erreurs commises par Winckelmann (Goethe et Herder 1993, p. 33), mais aussi des artistes eux-mêmes, dont certains s'en sont amusés. Le peintre Giovanni Battista Casanova (1730-1798), frère du célèbre aventurier, réalise ainsi deux fausses fresques antiques, représentant Trois Ménades dansant et une Athéna parmi les filles de Cécrops, que le savant allemand intègre ingénument dans la première édition de sa Geschichte (2005, p. 419-421). Mengs ira plus loin, en élaborant un stratagème plus complexe pour faire croire à son ami à l'authenticité de son Jupiter et Ganymède. Winckelmann décide sans hésiter d'intégrer cette œuvre au corpus de sa Geschichte, parce qu'elle est peinte en respectant ce que l'on pensait être les techniques des peintres antiques et les idées exprimées par Winckelmann sur la peinture antiques, mais aussi, sans doute, parce qu'elle justifie par l'image les opinions du savant sur les mœurs homosexuelles des Grecs et des Romains (Décultot 2000, p. 45) :

[C'est] une peinture d'une qualité telle qu'on n'en avait jamais vu jusque-là, éclipsant même les peintures d'Herculanum alors connues. Il s'agit d'un Jupiter assis, couronné de lierre (celui d'Élis avait une couronne de fleurs), s'apprêtant à embrasser Ganymède, qui lui tend, de la main droite, une coupe agrémentée d'ornements en relief et, de la main gauche, un récipient dans lequel il servait aux dieux de l'ambroisie. [...] Le favori de Jupiter est sans conteste une des plus belles figures que nous ait léguées l'Antiquité, et je ne trouve rien de comparable à son visage : une telle volupté s'épanouit sur ce visage que son expression entière semble n'être qu'un baiser (2005, p. 418-419).

70 Aucun de ces lecteurs, pourtant critiques, de Winckelmann n'a pour autant remis en cause la légitimité de son approche. La Grèce n'est pas, pour le savant allemand, une " période » de l'histoire mais, plutôt, une utopie servant aux Modernes à se raconter, en construisant, dans le temps et le passé, l'origine d'une grandeur perdue dont ils ne peuvent nullement être considérés comme les enfants ou les héritiers, mais qu'ils doivent apprendre à regretter. Avant d'être l'objet d'une histoire, le monde grec décrit par Johann Joachim Winckelmann apparaît donc comme une fiction, paradoxale à plusieurs égards, et, d'abord, dans la manière dont elle tente d'articuler son rapport au passé. En cela, elle est profondément moderne, et même, comme le souligne Herder, profondément "allemande »: "Winckelmann était allemand, et il le resta même à Rome. En Italie encore, il rédigea ses textes en allemand et pour l'Allemagne, et il éprouva toujours, du plus loin qu'il se trouvait, de l'amour pour ses compatriotes et sa patrie » (Goethe et Herder 1993, p. 7). La Grèce n'est pas arrachée à l'oubli comme un vase ou un fragment de marbre du sol ; comme les statues et les tableaux vus et décrits par Denis Diderot dans les Salons, elle est le produit d'une résonance ou d'un écho sensibles, en grande partie pris en 
charge par une langue qui, à la manière d'une ekphrasis, comble une absence de choses par une abondance de mots, comme le note Goethe :

Nous voyons Winckelmann lui-même écrire comme un poète, un poète dont on ne peut ignorer la grande valeur, quand il entreprend de décrire des statues. Il voit de ses propres yeux, il saisit de toute sa sensibilité des œuvres indicibles tout en éprouvant pourtant le besoin irrésistible d'en rendre compte par l'écriture (p. 100).

Plus que la restitution d'une mémoire, sortie de l'oubli, Winckelmann cherche, en antiquaire du $\mathrm{xVIII}^{\mathrm{e}}$ siècle, à générer "des lieux de mémoire dans la conscience des peuples de cette Europe » (Prost 2010, p. 68).

La Grèce telle qu'elle est inventée par Winckelmann tient donc, tout à la fois, des deux acceptions modernes de la notion de «civilisation». Voisine de celle de progrès (Starobinski 1983, p. 17), sa " civilisation " pose initialement et dans un passé, mythique et lointain, un état primitif de nature et de sauvagerie, dont la Grèce est censée s'être détachée ou sur laquelle elle a marqué son empreinte. Dans cette perspective, la civilisation est une norme idéologique, qui jette une double lumière sur l'histoire : celle, brillante, d'un progrès, mais qui n'est observable dans les restes et les ruines du passé ; et, donc, celle, plus inquiétante, d'une décadence inéluctable, associée, notamment pendant et après la Querelle des Anciens et des Modernes, aux thèmes de la corruption du goût et des mœurs (Lecoq 2001), ou encore à la «fausse civilisation » ou à la «barbarie de nos civilisations » raillées par Mirabeau (Starobinski 1983, p. 20). Winckelmann explique ainsi que la responsabilité des historiens n'est pas seulement de dire le vrai sur le passé, mais de donner à ce passé la possibilité de survivre dans le souvenir, malgré la ruine et la destruction. C'est la raison d'être des dernières lignes de la préface de la Geschichte: "Cette histoire de l'art, je la dédie à l'art, au temps, et plus particulièrement à mon ami Anton Raphaël Mengs » (Décultot 2000, p. 68). Tandis que les artistes, dont Mengs est présenté comme le meilleur représentant, font partie de ceux auxquels Winckelmann souhaite particulièrement s'adresser, le savant fait amende honorable devant la grandeur infinie de l'art antique et la puissance irrésistible du temps.

\section{BIBLIOGRAPHIE}

Benveniste É. (1966), Problèmes de linguistique générale, Paris.

Bert J.-F. (2009), « Marcel Mauss et la notion de "civilisation" ", Cahiers de recherche sociologique 47, p. 123-142.

Blanc J. (2016). « "Un plaisir intellectuel” : Sir Joshua Reynolds et le refus de la sculpture peinte » dans Extermann G. et Varela Braga A., Splendor marmoris : $i$ colori del marmo, tra Roma e l'Europa, da Paolo III a Napoleone III, Rome, p. 401-418.

Braudel F. (1990), La Méditerranée et le monde méditerranéen à l'époque de Philippe II, Paris.

Brinkmann V. et Kunze M. (2011), Die Artemis von Pompeji und die Entdeckung der Farbigkeit griechischer Plastik, Mayence. 
Calame C. (1996), «Le mythe, une catégorie hellène ", Recherches sur la philosophie et le langage 18, p. 85-107.

- (2000), Poétique des mythes dans la Grèce antique, Paris.

Compagnon A. (2005), Les Antimodernes, de Joseph de Maistre à Roland Barthes, Paris.

Décultot É. (2000), Johann Joachim Winckelmann : enquête sur la genèse de l'histoire de l'art, Paris.

Diderot D. (2000), Euvres : Esthétique, théâtre, éd. L. Versini, Paris.

Dupont F. (2011), Rome, la ville sans origine : l'Énéide, un grand récit du métissage ?, Paris.

Febvre L., Mauss M., Tonnelat É. et Niceforo A. (1930), Civilisation : le mot et l'idée, Paris.

Flavell M. K. (1979), « Winckelmann and the German Enlightenment: On the Recovery and Uses of the Past », The Modern Language Review 76-1, p. 79-96.

Furetière A. (1690), Dictionnaire universel, contenant généralement tous les mots français tant vieux que modernes, et les termes de toutes les sciences et des arts, 3 vol., La Haye-Rotterdam.

Gauvard C. et Sirinelli J.-F. éd. (2015), Dictionnaire de l'historien, Paris.

Ginzburg C. et Castelnuovo E. (1981), « Domination symbolique et géographie artistique dans l'histoire de l'art italien ", Actes de la recherche en sciences sociales 40, 1, p. 51-72.

Goethe J. W. von et Herder J. G. (1993), Le Tombeau de Winckelmann, trad. M. Charrière-Jacquin, Nîmes.

Heres G. (1991), Winckelmann in Sachsen : ein Beitrag zur Kulturgeschichte Dresdens und zur Biographie Winckelmanns, Leipzig.

Howard S. (1990), Antiquity Restored: Essays on the Afterlife of the Antique, Vienne.

Kaibel G. (1890), Inscriptiones Italiae et Siciliae, éd. A. Lebègue, Berlin.

Kultermann U. (1993), The History of Art History, New York.

Lacoste Y. (2014), La Géographie, ça sert d'abord à faire la guerre, Paris.

Lecoq A.-M. éd. (2001), La Querelle des Anciens et des Modernes : XVII ${ }^{e}$-XVIII ${ }^{e}$ siècles, Paris.

Lichtenstein J. (1989), La Couleur éloquente : rhétorique et peinture à l'âge classique, Paris.

Macmillan D. éd. (1986), Painting in Scotland: The Golden Age, Oxford.

Morrison J. (1996), Winckelmann and the Notion of Aesthetic Education, Oxford.

Pomian K. (1999), Sur l'histoire, Paris.

Pommier E. (2003), Winckelmann, inventeur de l'histoire de l'art, Paris.

Potts A. (1982), « Winckelmann's Construction of History », Art History 5-4, p. 377-407.

- (1990), « The Verbal and Visual In Winckelmann's Analysis of Style », Word \& Image, 6-3,

p. 226-240.

Prost F. (2010), « Archéologie« », dans Delacroix C., Dosse F., Garcia P. et Offenstadt N. éd., Historiographies : concepts et débats, 2 vol., t. I, Paris, p. 54-67.

Seeba H. C. (1982), « Johann Joachim Winckelmann Zur Wirkungsgeschichte eines

"unhistorischen" Historikers zwischen Ästhetik und Geschichte ", Deutsche Vierteljahrsschrift für Literaturwissenschaft und Geistesgeschichte 56-1, p. 168-201.

Starobinski J. (1983), « Le mot Civilisation », Le Temps de la réflexion, 4, p. 13-51. 
Vernant J.-P. (1962), Les Origines de la pensée grecque, Paris.

Voltaire (1805), Essai sur les mœurs, 8 vol., Paris (rééd. 1756).

Winckelmann J. J. (1952), Briefe, éd. W. Rehm, 4 vol., Berlin.

- (1968), Kleine Schriften, Vorreden, Entwürfe, éd. W. Rehm et H. Sichtermann, Berlin.

- (1991), Réflexions sur l'imitation des oeuvres grecques en peinture et en sculpture, trad. M. CharrièreJacquin, Nîmes.

- (2005), Histoire de l'art dans l'Antiquité, éd. D. Gallo, trad. D. Tassel, Paris.

\section{NOTES}

1. «The idealization of the Greek world and its art is one of the most striking ahistorical aspects of the theoretical work of the period» (Morrison 1996, p. 23).

2. «It may be said of Homer, and of every poet who has wrote [sic] well that what he felt and saw that he described: had he been born much sooner he would have seen nothing but nakedness and barbarity » (Macmillan 1986, p. 39). Sur la lecture de lord Shaftesbury et de Thomas Blackwell par Winckelmann, voir Décultot 2000, p. 143-146.

3. Pour une déconstruction de cette notion, voir notamment Vernant 1962.

4. Pour une analyse simpliste des rapports entre art et territoires, voir Ginzburg et Castelnuovo 1981. Pour une vision plus nuancée des problèmes posés par les modèles géographiques, voir notamment Lacoste 2014.

5. « Not only the individual advances from infancy to manhood, but the species itself from rudeness to civilisation » (History of Civil Society, 1767), cité par Benveniste 1966, p. 336-345.

\section{RÉSUMÉS}

Souvent considéré comme le premier historien de l'art et comme le précurseur des développements de l'archéologie moderne, Johann Joachim Winckelmann (1717-1768) est aussi, et peut-être d'abord, l'un des derniers mythographes de l'Antiquité. Ses écrits construisent en effet la fiction d'une Grèce dont les principales caractéristiques correspondent à celles qui seront assignées à la notion de civilisation durant le dernier tiers du XVIII ${ }^{\mathrm{e}}$ siècle. Pour Winckelmann, qui la situe dans un passé à la fois lointain et mythique, la Grèce s'est construite après et contre un état primitif de nature et de sauvagerie, qu'elle a su dépasser. Dans ce cadre, la responsabilité des historiens et des artistes n'est pas seulement de dire le vrai sur le passé de la Grèce, mais de donner à ce passé la possibilité de survivre dans le souvenir, malgré la ruine et la destruction propres au monde moderne.

Johann Joachim Winckelmann (1717-1768) is often regarded as the first art historian and the precursor of modern archaeology. Yet he is also, and perhaps first of all, one of the last mythographers of Antiquity. His writings indeed construct the fiction of a Greece whose main 
characteristics correspond to those which will be assigned to the notion of civilization during the last third of the 18th century. For Winckelmann, who situates it in a remote and mythical past, Greece was built after and against a primitive state of nature and savagery, which it overcame. In this context, the responsibility of historians and artists is not only to tell the truth about Greece's past, but to give the past the opportunity to survive in remembrance, despite the ruin and destruction of the modern world.

INDEX

Keywords : history, art history, archaeology, civilization, Greece, Winckelmann

Mots-clés : histoire, histoire de l'art, archéologie, civilisation, Grèce, Winckelmann, Antiquité

\section{AUTEUR}

\section{JAN BLANC}

Université de Genève - jan.blanc@unige.ch 